Annals of Pure and Applied Mathematics

Vol. 16, No. 2, 2018, 255-263

ISSN: 2279-087X (P), 2279-0888(online)

Published on 1 February 2018

www.researchmathsci.org

DOI: http://dx.doi.org/10.22457/apam.v16n2al

Annals of

Pure and Applied

Mathematics

\title{
One Modulo Three Root Square Mean Labeling of Some Disconnected Graphs
}

\author{
M. Jaslin Melbha ${ }^{1}$ and C. Jayasekaran ${ }^{2}$ \\ ${ }^{1}$ Department of Mathematics, Women's Christian College \\ Nagercoil - 629001, TamilNadu, India. Email: mjaslinmelbha@gmail.com \\ ${ }^{2}$ Department of Mathematics, Pioneer Kumaraswamy College \\ Nagercoil - 629003, TamilNadu, India. Email: jaya_pkc@yahoo.com
}

Received 10 January 2018; accepted 28 January 2018

Abstract. A graph $G$ is said to be one modulo three root square mean graph if there is an injective function $\varphi$ from the vertex set of $G$ to the set $\{0,1,3, \ldots, 3 q-2,3 q\}$ where $q$ is the number of edges of $G$ and $\varphi$ induces a bijection $\varphi^{*}$ from the edge set of $G$ to $\{1,4, \ldots$, $3 q-2\}$ given by $\varphi^{*}(\mathrm{uv})=\left\lceil\sqrt{\frac{[\varphi(u)]^{2}+[\varphi(v)]^{2}}{2}}\right\rceil$ or $\left\lfloor\sqrt{\frac{[\varphi(u)]^{2}+[\varphi(v)]^{2}}{2}}\right\rfloor$ and the function $\varphi$ is called one modulo three root square mean labeling of $G$. The concept of one modulo three root square mean labeling was introduced by Jayasekaran and Jaslin Melbha and they investigated some graphs are one modulo three root square mean graphs. In this paper we prove that some disconnected graphs are one modulo three root square mean labeling.

Keywords: one modulo three root square mean labeling, one modulo three root square mean graphs.

\section{AMS Mathematics Subject Classification (2010): 05C99, 05 C22}

\section{Introduction}

We begin with simple, finite, connected and undirected graph. For standard terminology and notations we follow Harary [1]. A graph labeling is an assignment of integers to the vertices or edges or both subject to certain condition(s). If the domain of the mapping is the set of vertices (edges) then the labeling is called a vertex labeling (an edge labeling). Several types of graph labeling and a detailed survey are available in [2]. A very good application of graph labeling is given in [3,4]

Jayasekaran and David Raj introduced the concept one modulo three harmonic mean labeling of graphs in [5]. Root square mean labeling was introduced by Sandhya, Somasundaram and Anusa in [6]. Jayasekaran and Jaslin Melbha introduced the concept one modulo three root square mean labeling of graphs in [7]. Further they investigated some graphs are one modulo three root square mean graphs [8]. Not every graph is one modulo three root square mean. For example, star graph $K_{1, n}$, where $n \geq 4$ is not a one modulo three root square mean graph. We are interested to study different classes of graphs, which are one modulo three root square mean graphs. 


\section{Jaslin Melbha and C. Jayasekaran}

We will provide a brief summary of definitions and other information's which are necessary for our present investigation.

Definition 1.1. A graph $G$ is said to be one modulo three root square mean graph if there is an injective function $\varphi$ from the vertex set of $G$ to the set $\{0,1,3, \ldots, 3 q-2,3 q\}$ where $q$ is the number of edges of $G$ and $\varphi$ induces a bijection $\varphi^{*}$ from the edge set of $\mathrm{G}$ to $\{1,4$, $\ldots, 3 q-2\}$ given by $\varphi^{*}(\mathrm{uv})=\left[\sqrt{\frac{[\varphi(u)]^{2}+[\varphi(v)]^{2}}{2}}\right\rceil$ or $\left\lfloor\sqrt{\frac{[\varphi(u)]^{2}+[\varphi(v)]^{2}}{2}}\right\rfloor$ and the function $\varphi$ is called one modulo three root square mean labeling of $G$.

Definition 1.2. The corona of two graphs $G_{1}$ and $G_{2}$ is the graph $G=G_{1} \odot G_{2}$ formed from one copy of $G_{1}$ and $\left|V\left(G_{1}\right)\right|$ copies of $G_{2}$ where $i^{\text {th }}$ vertex of $G_{1}$ is adjacent to every vertices in the $i^{\text {th }}$ copy of $G_{2}$.

Definition 1.3. The graph $\mathrm{P}_{\mathrm{n}} \odot \mathrm{K}_{1}$ is called a comb.

Definition 1.4. The product $\mathrm{P}_{2} \times \mathrm{P}_{\mathrm{n}}$ is called a ladder and it is denoted by $\mathrm{L}_{\mathrm{n}}$. The ladder graph $L_{n}$ is a planar undirected graph with $2 n$ vertices and $3 n-2$ edges.

Definition 1.5. The union of two graphs $G_{1}=\left(V_{1}, E_{1}\right)$ and $G_{2}=\left(V_{2}, E_{2}\right)$ is a graph $G=$ $G_{1} \cup G_{2}$ with vertex set $V=V_{1} \cup V_{2}$ and the edge set $E=E_{l} \cup E_{2}$.

Theorem 1.6. The ladder $L_{n}$ is a one modulo three root square mean graph for $\mathrm{n} \neq 2[6]$.

Theorem 1.7. $P_{m} \cup\left(P_{n} \odot K_{l}\right)$ is a one modulo three root square mean graph [6].

\section{Main results}

Theorem 2.1. $\left(L_{m} \odot K_{l}\right) \cup P_{n}$ is one modulo three root square mean graph.

Proof: Let $u_{1} u_{2} \ldots u_{m}$ and $v_{1} v_{2} \ldots v_{m}$ be two paths of length $m$. Join $u_{i}$ and $v_{i}, 1 \leq i \leq m$. The resultant graph is $\mathrm{L}_{\mathrm{m}}$. For $1 \leq \mathrm{i} \leq \mathrm{m}$, let $\mathrm{x}_{\mathrm{i}}$ be the pendant vertex adjacent to $\mathrm{u}_{\mathrm{i}}$ and $\mathrm{y}_{\mathrm{i}}$ be the pendant vertex adjacent to $\mathrm{v}_{\mathrm{i}}$. Then we get the graph $L_{m} \odot K_{l}$. Let $\mathrm{w}_{1} \mathrm{w}_{2} \ldots \mathrm{w}_{\mathrm{n}}$ be the path $\mathrm{P}_{\mathrm{n}}$. Let $G=\left(L_{m} \odot K_{l}\right) \cup P_{n}$ with $V(\mathrm{G})=\left\{\mathrm{u}_{\mathrm{i}}, \mathrm{v}_{\mathrm{i}}, \mathrm{x}_{\mathrm{i}}, \mathrm{y}_{\mathrm{i}}, \mathrm{w}_{\mathrm{j}} / 1 \leq \mathrm{i} \leq \mathrm{m}, 1 \leq \mathrm{j} \leq \mathrm{n}\right\}$ and $E(\mathrm{G})=$ $\left\{\mathrm{u}_{\mathrm{i}} \mathrm{v}_{\mathrm{i}}, \mathrm{u}_{\mathrm{i}} \mathrm{x}_{\mathrm{i}}, \mathrm{v}_{\mathrm{i}} \mathrm{y}_{\mathrm{i}}, \mathrm{v}_{\mathrm{j}} \mathrm{v}_{\mathrm{j}+1}, \mathrm{u}_{\mathrm{j}} \mathrm{u}_{\mathrm{j}+1}, \mathrm{w}_{\mathrm{j}} \mathrm{w}_{\mathrm{j}+1} / 1 \leq \mathrm{i} \leq \mathrm{m}, 1 \leq \mathrm{j} \leq \mathrm{n}-1\right\}$. Then $\mathrm{G}$ has $4 \mathrm{~m}+\mathrm{n}$ vertices and $5 \mathrm{~m}+\mathrm{n}-3$ edges. Define a function $\phi: V(G) \rightarrow\{0,1,3, \ldots, 3 q-2,3 q\}$ by $\phi\left(\mathrm{u}_{1}\right)=1, \phi\left(\mathrm{u}_{2}\right)=$ $15, \phi\left(\mathrm{u}_{3}\right)=33, \phi\left(\mathrm{u}_{4}\right)=46, \phi\left(\mathrm{u}_{\mathrm{i}}\right)=15 \mathrm{i}-12,5 \leq \mathrm{i} \leq \mathrm{m} ; \phi\left(\mathrm{x}_{1}\right)=0, \phi\left(\mathrm{x}_{2}\right)=22, \phi\left(\mathrm{x}_{3}\right)=28$, $\phi\left(\mathrm{x}_{4}\right)=45, \phi\left(\mathrm{x}_{\mathrm{i}}\right)=15 \mathrm{i}-17,5 \leq \mathrm{i} \leq \mathrm{m} ; \phi\left(\mathrm{v}_{1}\right)=6, \phi\left(\mathrm{v}_{2}\right)=18, \phi\left(\mathrm{v}_{3}\right)=36, \phi\left(\mathrm{v}_{\mathrm{i}}\right)=15 \mathrm{i}-9,4 \leq \mathrm{i}$ $\leq \mathrm{m} ; \phi\left(\mathrm{y}_{1}\right)=9, \phi\left(\mathrm{y}_{2}\right)=24, \phi\left(\mathrm{y}_{3}\right)=39, \phi\left(\mathrm{y}_{\mathrm{i}}\right)=15 \mathrm{i}-8,4 \leq \mathrm{i} \leq \mathrm{m} ; \phi\left(\mathrm{w}_{\mathrm{i}}\right)=15 \mathrm{~m}+3 \mathrm{i}-9,1 \leq \mathrm{i} \leq \mathrm{n}$. Then $\phi$ induces a bijection $\phi^{*}: E(G) \rightarrow\{1,4, \ldots, 3 q-2\}$, where $\phi^{*}\left(\mathrm{u}_{\mathrm{i}} \mathrm{u}_{\mathrm{i}+1}\right)=15 \mathrm{i}-5,1 \leq \mathrm{i} \leq$ $\mathrm{m}-1 ; \phi^{*}\left(\mathrm{u}_{1} \mathrm{x}_{1}\right)=1, \phi^{*}\left(\mathrm{u}_{2} \mathrm{x}_{2}\right)=19, \phi^{*}\left(\mathrm{u}_{\mathrm{i}} \mathrm{x}_{\mathrm{i}}\right)=15 \mathrm{i}-14,3 \leq \mathrm{i} \leq \mathrm{m} ; \phi^{*}\left(\mathrm{u}_{1} \mathrm{v}_{1}\right)=4, \phi^{*}\left(\mathrm{u}_{2} \mathrm{v}_{2}\right)=16$, $\phi^{*}\left(\mathrm{u}_{\mathrm{i}} \mathrm{v}_{\mathrm{i}}\right)=15 \mathrm{i}-11,3 \leq \mathrm{i} \leq \mathrm{m} ; \phi^{*}\left(\mathrm{v}_{1} \mathrm{y}_{1}\right)=7, \phi^{*}\left(\mathrm{v}_{2} \mathrm{y}_{2}\right)=22, \phi^{*}\left(\mathrm{v}_{\mathrm{i}} \mathrm{y}_{\mathrm{i}}\right)=15 \mathrm{i}-8,3 \leq \mathrm{i} \leq \mathrm{m} ;$ $\phi^{*}\left(\mathrm{v}_{\mathrm{i}} \mathrm{v}_{\mathrm{i}+1}\right)=15 \mathrm{i}-2,1 \leq \mathrm{i} \leq \mathrm{m}-1 ; \phi^{*}\left(\mathrm{w}_{\mathrm{i}} \mathrm{w}_{\mathrm{i}+1}\right)=15 \mathrm{~m}+3 \mathrm{i}-8,1 \leq \mathrm{i} \leq \mathrm{n}-1$. Hence $\left(L_{m} \odot K_{l}\right) \cup P_{n}$ is one modulo three root square mean graph.

Example 2.2. A one modulo three root square mean labeling of $\left(L_{8} \odot K_{1}\right) \cup P_{7}$ is given in figure 1. 
One Modulo Three Root Square Mean Labeling of Some Disconnected Graphs

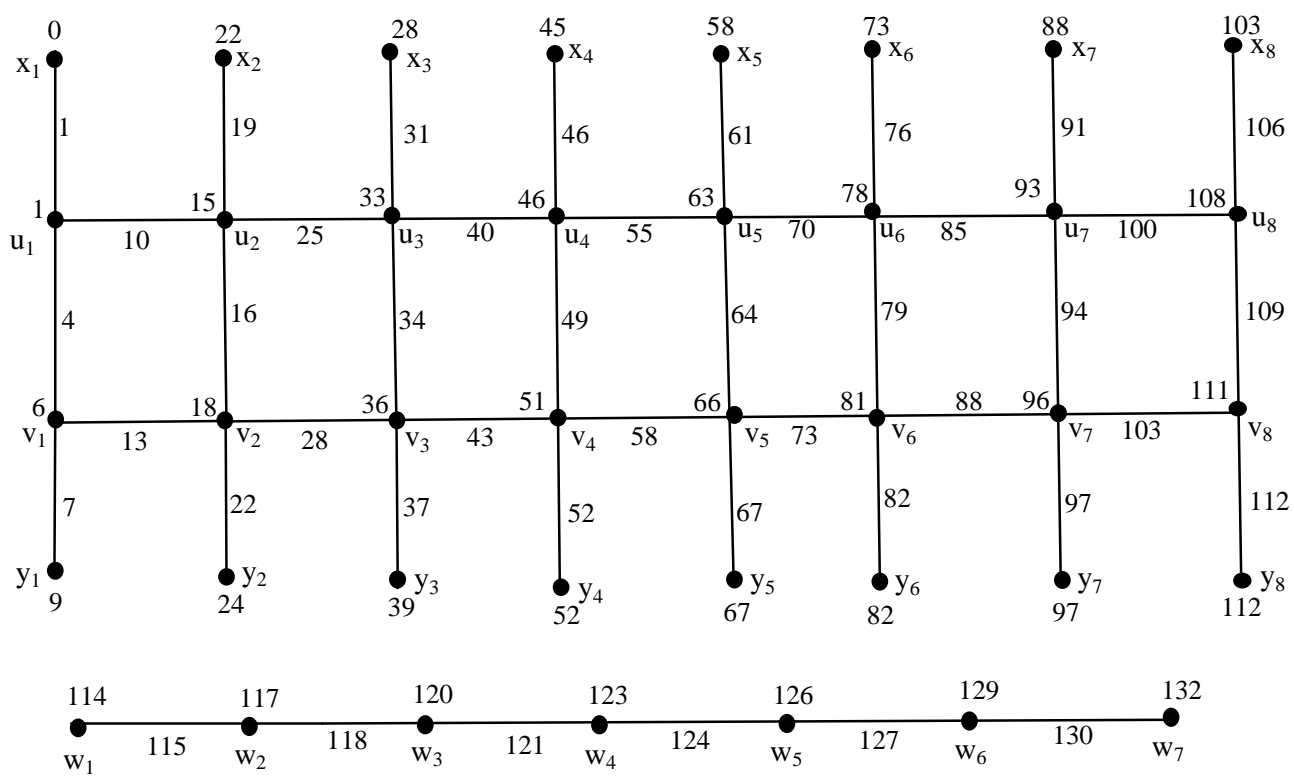

Figure 1: $\left(L_{8} \odot K_{1}\right) \cup P_{7}$

Theorem 2.3. $\left(L_{m} \odot K_{l}\right) \cup\left(P_{n} \odot K_{l}\right)$ is one modulo three root square mean graph.

Proof: Let $\mathrm{u}_{1} \mathrm{u}_{2} \ldots \mathrm{u}_{\mathrm{m}}$ and $\mathrm{v}_{1} \mathrm{v}_{2} \ldots \mathrm{v}_{\mathrm{m}}$ be two paths of length $\mathrm{m}$. Join $\mathrm{u}_{\mathrm{i}}$ and $\mathrm{v}_{\mathrm{i}}, 1 \leq \mathrm{i} \leq \mathrm{m}$. The resultant graph is $\mathrm{L}_{\mathrm{m}}$. For $1 \leq \mathrm{i} \leq \mathrm{m}$, let $\mathrm{x}_{\mathrm{i}}$ be the pendant vertex adjacent to $\mathrm{u}_{\mathrm{i}}$ and $\mathrm{y}_{\mathrm{i}}$ be the pendant vertex adjacent to $\mathrm{v}_{\mathrm{i}}$. Then we get the graph $L_{m} \odot K_{l}$. Let $\mathrm{P}_{\mathrm{n}}$ be the path $\mathrm{v}_{1} \mathrm{v}_{2} \ldots \mathrm{v}_{\mathrm{n}}$. Let $\mathrm{w}_{\mathrm{i}}$ be the vertex adjacent to $\mathrm{v}_{\mathrm{i}}, 1 \leq \mathrm{i} \leq \mathrm{n}$. The resultant graph is $\mathrm{P}_{\mathrm{n}} \odot \mathrm{K}_{1}$. Let $G=\left(L_{m}\right.$ $\left.\odot K_{l}\right) \cup\left(P_{n} \odot K_{l}\right)$ with $V(\mathrm{G})=\left\{\mathrm{u}_{\mathrm{i}}, \mathrm{v}_{\mathrm{i}}, \mathrm{x}_{\mathrm{i}}, \mathrm{y}_{\mathrm{i}}, \mathrm{s}_{\mathrm{j}}, \mathrm{t}_{\mathrm{j}} / 1 \leq \mathrm{i} \leq \mathrm{m}, 1 \leq \mathrm{j} \leq \mathrm{n}\right\}$ and $E(\mathrm{G})=\left\{\mathrm{u}_{\mathrm{i}} \mathrm{v}_{\mathrm{i}}, \mathrm{u}_{\mathrm{i}} \mathrm{x}_{\mathrm{i}}\right.$, $\left.\mathrm{v}_{\mathrm{i}} \mathrm{y}_{\mathrm{i}}, \mathrm{v}_{\mathrm{j}} \mathrm{v}_{\mathrm{j}+1}, \mathrm{u}_{\mathrm{j}} \mathrm{u}_{\mathrm{j}+1}, \mathrm{~s}_{\mathrm{j}} \mathrm{t}_{\mathrm{j}}, \mathrm{s}_{\mathrm{j}} \mathrm{s}_{\mathrm{j}+1} / 1 \leq \mathrm{i} \leq \mathrm{m}, 1 \leq \mathrm{j} \leq \mathrm{n}-1\right\}$. Then $\mathrm{G}$ has $4 \mathrm{~m}+2 \mathrm{n}$ vertices and $5 \mathrm{~m}+2 \mathrm{n}-3$ edges. Define a function $\phi: V(G) \rightarrow\{0,1,3, \ldots, 3 q-2,3 q\}$ by $\phi\left(\mathrm{u}_{1}\right)=1, \phi\left(\mathrm{u}_{2}\right)=15, \phi\left(\mathrm{u}_{3}\right)=$ 33, $\phi\left(\mathrm{u}_{4}\right)=46, \phi\left(\mathrm{u}_{\mathrm{i}}\right)=15 \mathrm{i}-12,5 \leq \mathrm{i} \leq \mathrm{m} ; \phi\left(\mathrm{x}_{1}\right)=0, \phi\left(\mathrm{x}_{2}\right)=22, \phi\left(\mathrm{x}_{3}\right)=28, \phi\left(\mathrm{x}_{4}\right)=45, \phi\left(\mathrm{x}_{\mathrm{i}}\right)=$ $15 \mathrm{i}-17,5 \leq \mathrm{i} \leq \mathrm{m} ; \phi\left(\mathrm{v}_{1}\right)=6, \phi\left(\mathrm{v}_{2}\right)=18, \phi\left(\mathrm{v}_{3}\right)=34, \phi\left(\mathrm{v}_{\mathrm{i}}\right)=15 \mathrm{i}-9,4 \leq \mathrm{i} \leq \mathrm{m} ; \phi\left(\mathrm{y}_{1}\right)=9, \phi\left(\mathrm{y}_{2}\right)$ $=24, \phi\left(\mathrm{y}_{3}\right)=39, \phi\left(\mathrm{y}_{\mathrm{i}}\right)=15 \mathrm{i}-8,4 \leq \mathrm{i} \leq \mathrm{m} ; \phi\left(\mathrm{s}_{\mathrm{i}}\right)=15 \mathrm{~m}+6 \mathrm{i}-9$ for all odd $\mathrm{i} \leq \mathrm{n} ; \phi\left(\mathrm{s}_{\mathrm{i}}\right)=15 \mathrm{~m}+6 \mathrm{i}-$ 12 for all even $\mathrm{i} \leq \mathrm{n} ; \phi\left(\mathrm{t}_{\mathrm{i}}\right)=15 \mathrm{~m}+6 \mathrm{i}-12$ for all odd $\mathrm{i} \leq \mathrm{n} ; \phi\left(\mathrm{t}_{\mathrm{i}}\right)=15 \mathrm{~m}+6 \mathrm{i}-9$ for all even $\mathrm{i} \leq$ $\mathrm{n}$. Then $\phi$ induces a bijection $\phi^{*}: E(G) \rightarrow\{1,4, \ldots, 3 q-2\}$, where $\phi^{*}\left(\mathrm{u}_{\mathrm{i}} \mathrm{u}_{\mathrm{i}+1}\right)=15 \mathrm{i}-5,1 \leq \mathrm{i} \leq$ $\mathrm{m}-1 ; \phi^{*}\left(\mathrm{u}_{1} \mathrm{x}_{1}\right)=1, \phi^{*}\left(\mathrm{u}_{2} \mathrm{x}_{2}\right)=19, \phi^{*}\left(\mathrm{u}_{\mathrm{i}} \mathrm{x}_{\mathrm{i}}\right)=15 \mathrm{i}-14,3 \leq \mathrm{i} \leq \mathrm{m} ; \phi^{*}\left(\mathrm{u}_{1} \mathrm{v}_{1}\right)=4, \phi^{*}\left(\mathrm{u}_{2} \mathrm{v}_{2}\right)=16$, $\phi^{*}\left(\mathrm{u}_{\mathrm{i}} \mathrm{v}_{\mathrm{i}}\right)=15 \mathrm{i}-11,3 \leq \mathrm{i} \leq \mathrm{m} ; \phi^{*}\left(\mathrm{v}_{1} \mathrm{y}_{1}\right)=7, \phi^{*}\left(\mathrm{v}_{2} \mathrm{y}_{2}\right)=22, \phi^{*}\left(\mathrm{v}_{\mathrm{i}} \mathrm{y}_{\mathrm{i}}\right)=15 \mathrm{i}-8,3 \leq \mathrm{i} \leq \mathrm{m} ;$ $\phi^{*}\left(\mathrm{v}_{\mathrm{i}} \mathrm{v}_{\mathrm{i}+1}\right)=15 \mathrm{i}-2,1 \leq \mathrm{i} \leq \mathrm{m}-1 ; \phi^{*}\left(\mathrm{~s}_{\mathrm{i}} \mathrm{t}_{\mathrm{i}}\right)=15 \mathrm{~m}+6 \mathrm{i}-11,1 \leq \mathrm{i} \leq \mathrm{n} ; \phi^{*}\left(\mathrm{~s}_{\mathrm{i}} \mathrm{s}_{\mathrm{i}+1}\right)=15 \mathrm{~m}+6 \mathrm{i}-8,1 \leq \mathrm{i} \leq$ n-1. Hence $\left(L_{m} \odot K_{l}\right) \cup\left(P_{n} \odot K_{l}\right)$ is one modulo three root square mean graph.

Example 2.4. A one modulo three root square mean labeling of $\left(L_{8} \odot K_{l}\right) \cup\left(P_{6} \odot K_{l}\right)$ is given in figure 2 . 
M. Jaslin Melbha and C. Jayasekaran
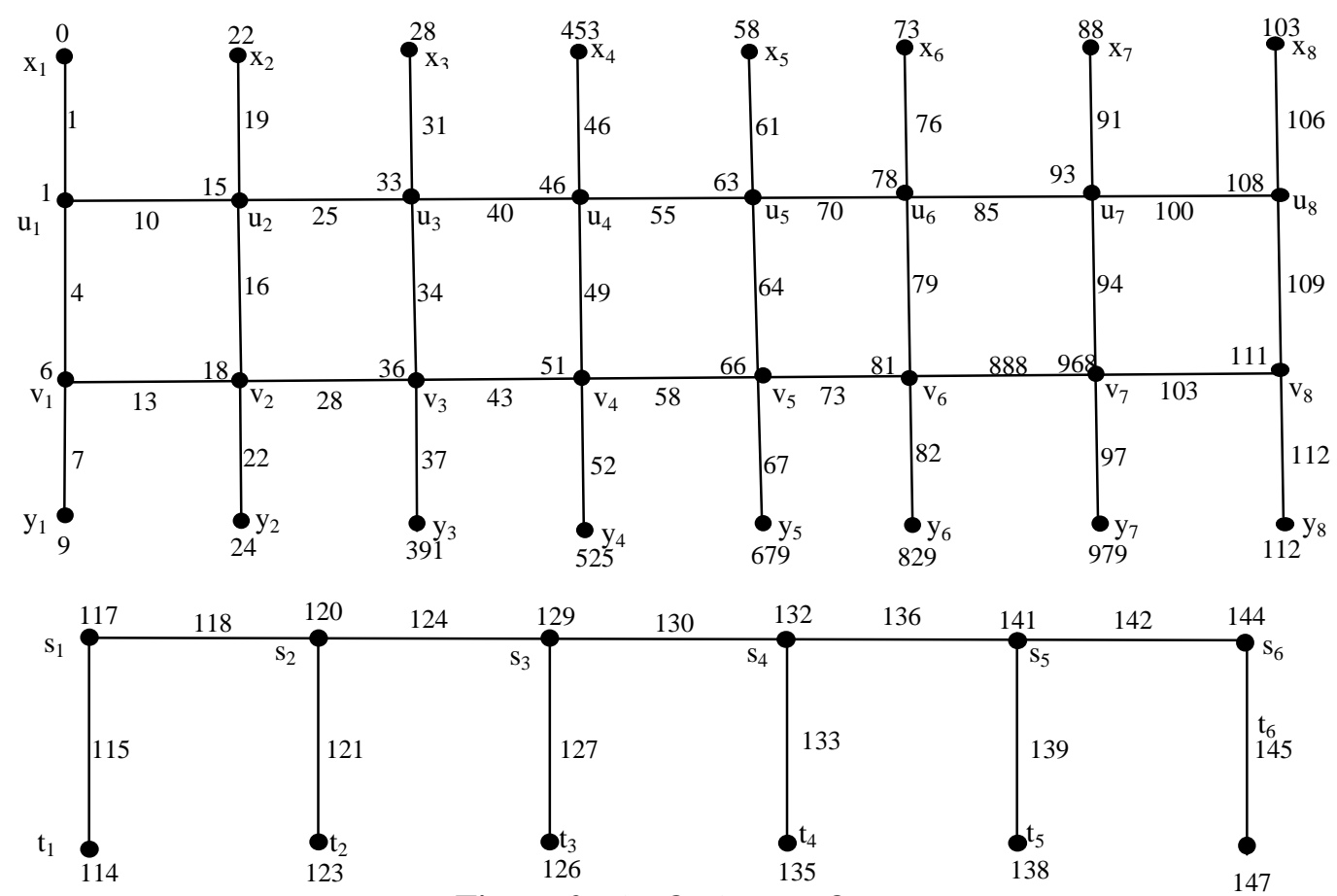

Figure 2: $\left(L_{\delta} \odot K_{l}\right) \cup\left(P_{6} \odot K_{l}\right)$

Theorem 2.5. $P_{n} \cup P_{m}$ is a one modulo three root square mean graph.

Proof: Let $\mathrm{u}_{1} \mathrm{u}_{2} \ldots \mathrm{u}_{\mathrm{n}}$ be the path $P_{n}$ and $\mathrm{v}_{1} \mathrm{v}_{2} \ldots \mathrm{v}_{\mathrm{m}}$ be the path $P_{m}$. Let $G=P_{n} \cup P_{m}$ with $V(G)=\left\{\mathrm{u}_{\mathrm{i}}, \mathrm{v}_{\mathrm{j}} / 1 \leq \mathrm{i} \leq \mathrm{n}, 1 \leq \mathrm{j} \leq \mathrm{m}\right\}$ and $E(G)=\left\{\mathrm{u}_{\mathrm{i}} \mathrm{u}_{\mathrm{i}+1}, \mathrm{v}_{\mathrm{j}} \mathrm{v}_{\mathrm{j}+1} / 1 \leq \mathrm{i} \leq \mathrm{n}-1,1 \leq \mathrm{j} \leq \mathrm{m}-1\right\}$. Then $G$ has $\mathrm{n}+\mathrm{m}$ vertices and $\mathrm{n}+\mathrm{m}-2$ edges. Define a function $\phi: V(G) \rightarrow\{0,1,3, \ldots, 3 q-2,3 q\}$ by $\phi\left(\mathrm{u}_{1}\right)=0, \phi\left(\mathrm{u}_{2}\right)=1, \phi\left(\mathrm{u}_{\mathrm{i}}\right)=3(\mathrm{i}-1), 3 \leq \mathrm{i} \leq \mathrm{n}-1, \phi\left(\mathrm{u}_{\mathrm{n}}\right)=3 \mathrm{n}-5 ; \phi\left(\mathrm{v}_{\mathrm{j}}\right)=3 \mathrm{n}+3 \mathrm{j}-6,1 \leq \mathrm{j} \leq \mathrm{m}-$ $1, \phi\left(\mathrm{v}_{\mathrm{m}}\right)=3 \mathrm{n}+3 \mathrm{j}-8$. Then $\phi$ induces a bijection $\phi^{*}: E(G) \rightarrow\{1,4, \ldots, 3 q-2\}$, where $\phi^{*}\left(\mathrm{u}_{\mathrm{i}} \mathrm{u}_{\mathrm{i}+1}\right)=3 \mathrm{i}-2,1 \leq \mathrm{i} \leq \mathrm{n}-1 ; \phi^{*}\left(\mathrm{v}_{\mathrm{j}} \mathrm{v}_{\mathrm{j}+1}\right)=3 \mathrm{n}+3 \mathrm{j}-5,1 \leq \mathrm{j} \leq \mathrm{m}-1$. Hence $P_{n} \cup P_{m}$ is a one modulo three root square mean graph .

Example 2.6. A one modulo three root square mean labeling of $P_{7} \cup P_{6}$ is given in figure 3 .

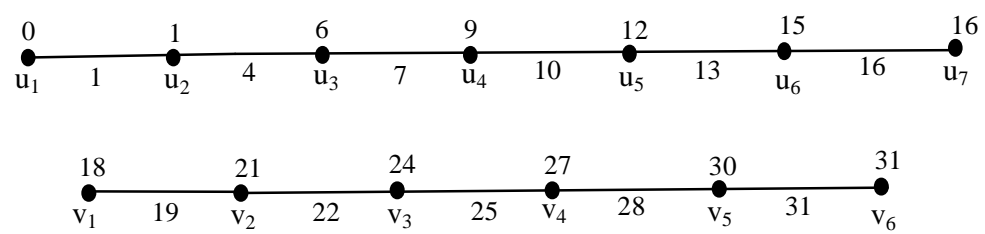

Figure 3: $P_{7} \cup P_{6}$

Theorem 2.7. $L_{m} \cup P_{n}$ is a one modulo three root square mean graph for $\mathrm{m} \neq 2$ and $\mathrm{n} \neq 3$. 
One Modulo Three Root Square Mean Labeling of Some Disconnected Graphs

Proof: Let $\mathrm{u}_{1} \mathrm{u}_{2} \ldots \mathrm{u}_{\mathrm{m}}$ and $\mathrm{v}_{1} \mathrm{v}_{2} \ldots \mathrm{v}_{\mathrm{m}}$ be two paths of length $\mathrm{m}$. Join $\mathrm{u}_{\mathrm{i}}$ and $\mathrm{v}_{\mathrm{i}}, 1 \leq \mathrm{i} \leq \mathrm{m}$. The resultant graph is $\mathrm{L}_{\mathrm{m}}$. Let $\mathrm{w}_{1} \mathrm{w}_{2} \ldots \mathrm{w}_{\mathrm{n}}$ be the path $\mathrm{P}_{\mathrm{n}}$. Let $G=L_{m} \cup P_{n}$ with $V(G)=\left\{\mathrm{u}_{\mathrm{i}}, \mathrm{v}_{\mathrm{i}}\right.$, $\left.\mathrm{w}_{\mathrm{j}} / 1 \leq \mathrm{i} \leq \mathrm{m}, 1 \leq \mathrm{j} \leq \mathrm{n}\right\}$ and $E(G)=\left\{\mathrm{u}_{\mathrm{i}} \mathrm{u}_{\mathrm{i}+1}, \mathrm{v}_{\mathrm{i}} \mathrm{v}_{\mathrm{i}+1}, \mathrm{u}_{\mathrm{i}} \mathrm{v}_{\mathrm{i}}, \mathrm{u}_{\mathrm{n}} \mathrm{v}_{\mathrm{n}}, \mathrm{w}_{\mathrm{j}} \mathrm{w}_{\mathrm{j}+1} / 1 \leq \mathrm{i} \leq \mathrm{m}-1,1 \leq \mathrm{j} \leq \mathrm{n}-1\right\}$. Then $G$ has $2 \mathrm{~m}+\mathrm{n}$ vertices and $3 \mathrm{~m}+\mathrm{n}-3$ edges.

Case 1. $m=1$

In this case, $L_{1} \cup P_{n}=P_{2} \cup P_{n}$. By Theorem 2.5 $P_{2} \cup P_{n}$ is a one modulo three root square mean graph.

Case 2. $m=2$

Sub case 2.1. $n=2$

In this case, $L_{m} \cup P_{n}=L_{2} \cup P_{2}$. Define a function $\phi: V(G) \rightarrow\{0,1,3, \ldots, 3 q-2,3 q\}$ by $\phi\left(\mathrm{u}_{1}\right)=3, \phi\left(\mathrm{u}_{2}\right)=4, \phi\left(\mathrm{v}_{1}\right)=15, \phi\left(\mathrm{v}_{2}\right)=10, \phi\left(\mathrm{w}_{1}\right)=0, \phi\left(\mathrm{w}_{2}\right)=1$. Then $\phi$ induces $\mathrm{a}$ bijection $\phi^{*}: E(G) \rightarrow\{1,4, \ldots, 3 q-2\}$, where $\phi^{*}\left(\mathrm{u}_{1} \mathrm{u}_{2}\right)=4, \phi^{*}\left(\mathrm{u}_{1} \mathrm{v}_{1}\right)=10, \phi^{*}\left(\mathrm{u}_{2} \mathrm{v}_{2}\right)=7$, $\phi^{*}\left(\mathrm{v}_{1} \mathrm{v}_{2}\right)=13, \phi^{*}\left(\mathrm{w}_{1} \mathrm{w}_{2}\right)=1$. Thus the edges get distinct labels $1,4, \ldots, 13$. In this case $\phi$ is a one modulo three root square mean labeling for $\mathrm{G}$. A one modulo three root square mean labeling of $L_{2} \cup P_{2}$ is given in figure 4 .
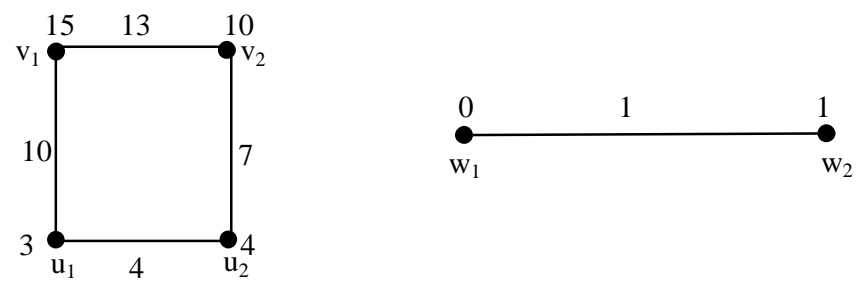

Figure 4: $L_{2} \cup P_{2}$

Sub case 2.2. $n=3$

In this case, $L_{m} \cup P_{n}=L_{2} \cup P_{3}$. It has 6 edges and 7 vertices. When we label these 7 vertices from the vertex set $\{0,1,3, \ldots, 18\}$, atleast two edges get the same labels and hence $L_{2} \cup P_{3}$ is not one modulo three root square mean labeling.

Sub case 2.3. $n \geq 4$

In this case, $L_{m} \cup P_{n}=L_{2} \cup P_{n}$. Define a function $\phi: V(G) \rightarrow\{0,1,3, \ldots, 3 q-2,3 q\}$ by $\phi\left(\mathrm{u}_{1}\right)=3 \mathrm{n}+9, \phi\left(\mathrm{u}_{2}\right)=3 \mathrm{n}+4 ; \phi\left(\mathrm{v}_{1}\right)=3 \mathrm{n}-3, \phi\left(\mathrm{v}_{2}\right)=3 \mathrm{n}-2 ; \phi\left(\mathrm{w}_{1}\right)=0, \phi\left(\mathrm{w}_{2}\right)=1, \phi\left(\mathrm{w}_{\mathrm{i}}\right)=3(\mathrm{i}-$ $1), 4 \leq \mathrm{i} \leq \mathrm{n}-1 ; \phi\left(\mathrm{w}_{\mathrm{n}}\right)=3 \mathrm{n}-5$. Then $\phi$ induces a bijection $\phi^{*}: E(G) \rightarrow\{1,4, \ldots, 3 q-2\}$, where $\phi^{*}\left(\mathrm{u}_{1} \mathrm{u}_{2}\right)=3 \mathrm{n}+7, \phi^{*}\left(\mathrm{u}_{2} \mathrm{v}_{2}\right)=3 \mathrm{n}+1, \phi^{*}\left(\mathrm{u}_{1} \mathrm{v}_{1}\right)=3 \mathrm{n}+4, \phi^{*}\left(\mathrm{v}_{1} \mathrm{v}_{2}\right)=3 \mathrm{n}-2 ; \phi^{*}\left(\mathrm{w}_{\mathrm{i}} \mathrm{w}_{\mathrm{i}+1}\right)=$ $3 \mathrm{i}-2,1 \leq \mathrm{i} \leq \mathrm{n}-1$. Thus the edges get distinct labels $1,4, \ldots, 3 \mathrm{q}-2$. In this case $\phi$ is a one modulo three root square mean labeling for $\mathrm{G}$.

Case 3. $m \geq 3$

Define a function $\phi: V(G) \rightarrow\{0,1,3, \ldots, 3 q-2,3 q\}$ by $\phi\left(\mathrm{u}_{1}\right)=0, \phi\left(\mathrm{u}_{2}\right)=1, \phi\left(\mathrm{u}_{3}\right)=6$, $\phi\left(\mathrm{u}_{\mathrm{i}}\right)=9 \mathrm{i}-6,4 \leq \mathrm{i} \leq \mathrm{m} ; \phi\left(\mathrm{v}_{\mathrm{i}}\right)=6 \mathrm{i}+3,1 \leq \mathrm{i} \leq 3 ; \phi\left(\mathrm{v}_{\mathrm{i}}\right)=9 \mathrm{i}-9,4 \leq \mathrm{i} \leq \mathrm{m} ; \phi\left(\mathrm{w}_{1}\right)=9 \mathrm{~m}-8, \phi\left(\mathrm{w}_{\mathrm{i}}\right)=$ $9(\mathrm{~m}-1)+3 \mathrm{i}, 2 \leq \mathrm{i} \leq \mathrm{n}$. Then $\phi$ induces a bijection $\phi^{*}: E(G) \rightarrow\{1,4, \ldots, 3 q-2\}$, where $\phi^{*}\left(\mathrm{u}_{1} \mathrm{u}_{2}\right)=1, \phi^{*}\left(\mathrm{u}_{2} \mathrm{u}_{3}\right)=4, \phi^{*}\left(\mathrm{u}_{3} \mathrm{u}_{4}\right)=22, \phi^{*}\left(\mathrm{u}_{\mathrm{i}} \mathrm{u}_{\mathrm{i}+1}\right)=9 \mathrm{i}-2,4 \leq \mathrm{i} \leq \mathrm{m}-1 ; \phi^{*}\left(\mathrm{v}_{\mathrm{i}} \mathrm{v}_{\mathrm{i}+1}\right)=6 \mathrm{i}+7,1 \leq$ $\mathrm{i} \leq 3 ; \phi^{*}\left(\mathrm{v}_{\mathrm{i}} \mathrm{v}_{\mathrm{i}+1}\right)=9 \mathrm{i}-5,4 \leq \mathrm{i} \leq \mathrm{m}-1 ; \phi^{*}\left(\mathrm{u}_{1} \mathrm{v}_{1}\right)=7, \phi^{*}\left(\mathrm{u}_{2} \mathrm{v}_{2}\right)=10, \phi^{*}\left(\mathrm{u}_{3} \mathrm{v}_{3}\right)=16, \phi^{*}\left(\mathrm{u}_{\mathrm{i}} \mathrm{v}_{\mathrm{i}}\right)=9 \mathrm{i}-$ $8,4 \leq \mathrm{i} \leq \mathrm{m} ; \phi^{*}\left(\mathrm{w}_{\mathrm{i}} \mathrm{W}_{\mathrm{i}+1}\right)=9 \mathrm{~m}+3 \mathrm{i}-8,1 \leq \mathrm{i} \leq \mathrm{n}-1$. Thus the edges get distinct labels $1,4, \ldots$, 


\section{Jaslin Melbha and C. Jayasekaran}

$3 \mathrm{q}-2$. In this case $\phi$ is a one modulo three root square mean labeling for G. Hence $L_{m} \cup P_{n}$ is a one modulo three root square mean graph for $m \neq 2$ and $n \neq 3$.

Example 2.8. A one modulo three root square mean labeling of $L_{2} \cup P_{4}$ and $L_{9} \cup P_{7}$ are shown in figure 5 and figure 6 respectively.
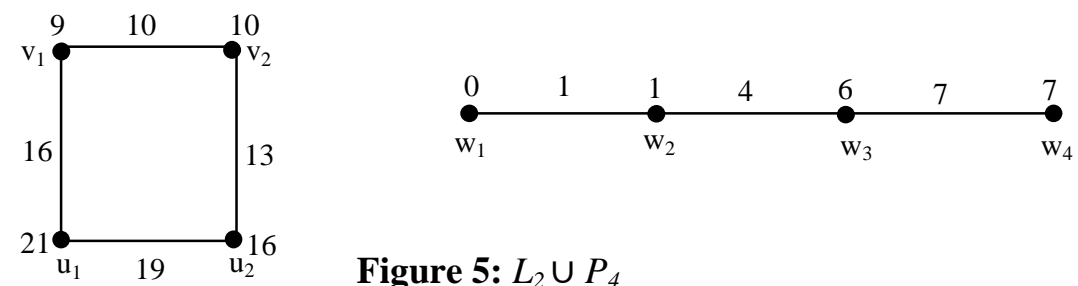

Figure 5: $L_{2} \cup P_{4}$
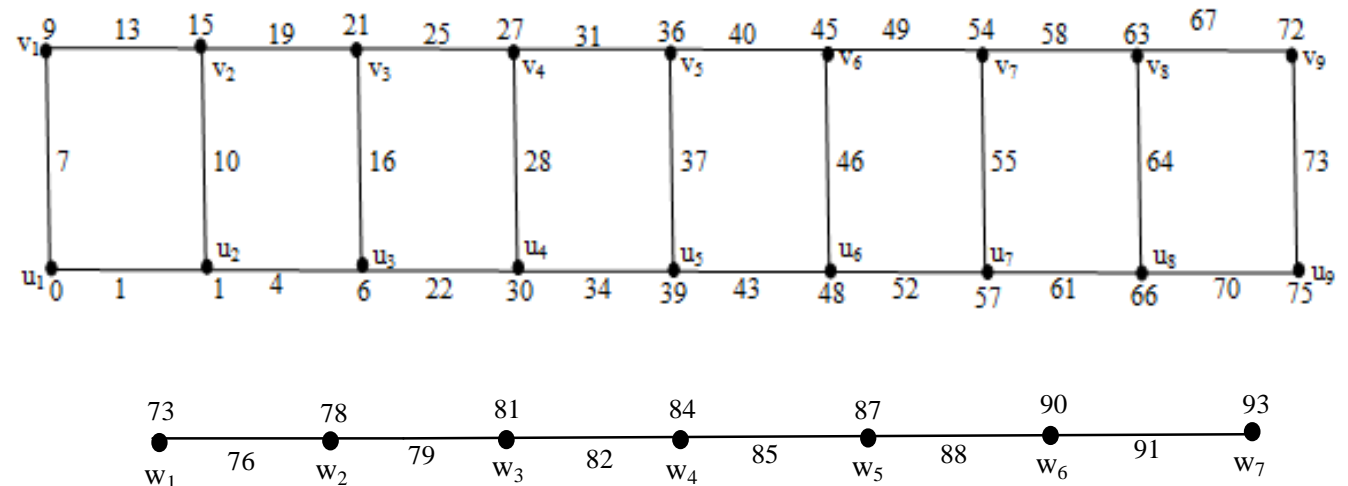

Figure 6: $L_{9} \cup P_{7}$

Theorem 2.9. $L_{m} \cup\left(P_{n} \odot K_{l}\right)$ is a one modulo three root square mean graph.

Proof: Let $\mathrm{u}_{1} \mathrm{u}_{2} \ldots \mathrm{u}_{\mathrm{m}}$ and $\mathrm{v}_{1} \mathrm{v}_{2} \ldots \mathrm{v}_{\mathrm{m}}$ be two paths of length $\mathrm{m}$. Join $\mathrm{u}_{\mathrm{i}}$ and $\mathrm{v}_{\mathrm{i}}, 1 \leq \mathrm{i} \leq \mathrm{m}$. The resultant graph is $\mathrm{L}_{\mathrm{m}}$. Let $\mathrm{s}_{1} \mathrm{~s}_{2} \ldots \mathrm{s}_{\mathrm{n}}$ be the path $\mathrm{P}_{\mathrm{n}}$. For $1 \leq \mathrm{i} \leq \mathrm{n}$, join $\mathrm{t}_{\mathrm{i}}$ with $\mathrm{s}_{\mathrm{i}}$. The resultant graph is $P_{n} \odot K_{l}$. Let $G=L_{m} \cup\left(P_{n} \odot K_{l}\right)$ with $V(G)=\left\{\mathrm{u}_{\mathrm{i}}, \mathrm{v}_{\mathrm{i}}, \mathrm{s}_{\mathrm{j}}, \mathrm{t}_{\mathrm{j}} / 1 \leq \mathrm{i} \leq \mathrm{m}, 1 \leq \mathrm{j} \leq \mathrm{n}\right\}$ and $E(G)=\left\{\mathrm{u}_{\mathrm{i}} \mathrm{u}_{\mathrm{i}+1}, \mathrm{v}_{\mathrm{i}} \mathrm{v}_{\mathrm{i}+1}, \mathrm{u}_{\mathrm{i}} \mathrm{v}_{\mathrm{i}}, \mathrm{u}_{\mathrm{m}} \mathrm{v}_{\mathrm{m}}, \mathrm{s}_{\mathrm{j}} \mathrm{s}_{\mathrm{j}+1}, \mathrm{~s}_{\mathrm{j}} \mathrm{t}_{j}, \mathrm{~s}_{\mathrm{n}} \mathrm{t}_{\mathrm{n}} / 1 \leq \mathrm{i} \leq \mathrm{m}-1,1 \leq \mathrm{j} \leq \mathrm{n}-1\right\}$. Then $G$ has $2 \mathrm{~m}+2 \mathrm{n}$ vertices and $3 m+2 n-3$ edges.

Case 1. $m=1$

In this case, $L_{l} \cup\left(P_{n} \odot K_{l}\right)=P_{2} \cup\left(P_{n} \odot K_{l}\right)$. By Theorem $1.7 P_{m} \cup\left(P_{n} \odot K_{l}\right)$ is a one modulo three root square mean graph.

Case 2. $\mathrm{m}=2$

Sub case 2.1. $n=1$

In this case, $L_{m} \cup\left(P_{n} \odot K_{1}\right)=L_{2} \cup P_{2}$. By Theorem 2.7, $L_{2} \cup P_{2}$ is a one modulo three root square mean graph.

Sub case 2.2. $n=2$

In this case, $L_{m} \cup\left(P_{n} \odot K_{l}\right)=L_{2} \cup P_{4}$. By Theorem 2.7, $L_{2} \cup P_{4}$ is a one modulo three root square mean graph. 
One Modulo Three Root Square Mean Labeling of Some Disconnected Graphs

Sub case 2.3. $n \geq 3$

In this case, $L_{m} \cup\left(P_{n} \odot K_{1}\right)=L_{2} \cup\left(P_{n} \odot K_{1}\right)$. Define a function $\phi: V(G) \rightarrow\{0,1,3, \ldots, 3 q$ $2,3 q\}$ by $\phi\left(\mathrm{u}_{1}\right)=6 \mathrm{n}+9, \phi\left(\mathrm{u}_{2}\right)=6 \mathrm{n}+4 ; \phi\left(\mathrm{v}_{1}\right)=6 \mathrm{n}-3, \phi\left(\mathrm{v}_{2}\right)=6 \mathrm{n}-2 ; \phi\left(\mathrm{s}_{1}\right)=0, \phi\left(\mathrm{s}_{\mathrm{i}}\right)=6 \mathrm{i}-5,2$ $\leq \mathrm{i} \leq \mathrm{n} ; \phi\left(\mathrm{t}_{1}\right)=1, \phi\left(\mathrm{t}_{\mathrm{i}}\right)=6 \mathrm{i}-6,2 \leq \mathrm{i} \leq \mathrm{n}$. Then $\phi$ induces a bijection $\phi^{*}: E(G) \rightarrow\{1,4, \ldots$, $3 q-2\}$, where $\phi^{*}\left(\mathrm{u}_{1} \mathrm{u}_{2}\right)=6 \mathrm{n}+7, \phi^{*}\left(\mathrm{u}_{2} \mathrm{v}_{2}\right)=6 \mathrm{n}+1, \phi^{*}\left(\mathrm{u}_{1} \mathrm{v}_{1}\right)=6 \mathrm{n}+4, \phi^{*}\left(\mathrm{v}_{1} \mathrm{v}_{2}\right)=6 \mathrm{n}-2$; $\phi^{*}\left(\mathrm{~s}_{\mathrm{i}} \mathrm{s}_{\mathrm{i}+1}\right)=6 \mathrm{i}-2,1 \leq \mathrm{i} \leq \mathrm{n}-1 ; \phi^{*}\left(\mathrm{~s}_{\mathrm{i}} \mathrm{t}_{\mathrm{i}}\right)=6 \mathrm{i}-5,1 \leq \mathrm{i} \leq \mathrm{n}$. Thus the edges get distinct labels $\{1,4$, $\ldots, 3 q-2\}$. In this case $\phi$ is a one modulo three root square mean labeling for G.

Case 3. $m \geq 3$

Define a function $\phi: V(G) \rightarrow\{0,1,3, \ldots, 3 q-2,3 q\}$ by $\phi\left(\mathrm{u}_{1}\right)=0, \phi\left(\mathrm{u}_{2}\right)=1, \phi\left(\mathrm{u}_{3}\right)=6$, $\phi\left(\mathrm{u}_{\mathrm{i}}\right)=9 \mathrm{i}-6,4 \leq \mathrm{i} \leq \mathrm{m} ; \phi\left(\mathrm{v}_{\mathrm{i}}\right)=6 \mathrm{i}+3,1 \leq \mathrm{i} \leq 3 ; \phi\left(\mathrm{v}_{\mathrm{i}}\right)=9(\mathrm{i}-1), 4 \leq \mathrm{i} \leq \mathrm{m} ; \phi\left(\mathrm{t}_{1}\right)=9 \mathrm{~m}-8, \phi\left(\mathrm{t}_{\mathrm{i}}\right)=$ $9 \mathrm{~m}+6 \mathrm{i}-12$ for all odd i, $3 \leq \mathrm{i} \leq \mathrm{n} ; \phi\left(\mathrm{t}_{\mathrm{i}}\right)=9 \mathrm{~m}+6 \mathrm{i}-9$ for all even $\mathrm{i} \leq \mathrm{n} ; \phi\left(\mathrm{s}_{\mathrm{i}}\right)=9 \mathrm{~m}+6 \mathrm{i}-9$ for all odd $\mathrm{i} \leq \mathrm{n} ; \phi\left(\mathrm{s}_{\mathrm{i}}\right)=9 \mathrm{~m}+6 \mathrm{i}-12$ for all even $\mathrm{i} \leq \mathrm{n}$. Then $\phi$ induces a bijection $\phi^{*}: E(G) \rightarrow\{1$, $4, \ldots, 3 q-2\}$, where $\phi^{*}\left(\mathrm{u}_{1} \mathrm{u}_{2}\right)=1, \phi^{*}\left(\mathrm{u}_{2} \mathrm{u}_{3}\right)=4, \phi^{*}\left(\mathrm{u}_{3} \mathrm{u}_{4}\right)=22, \phi^{*}\left(\mathrm{u}_{\mathrm{i}} \mathrm{u}_{\mathrm{i}+1}\right)=9 \mathrm{i}-2,4 \leq \mathrm{i} \leq \mathrm{m}-1$; $\phi^{*}\left(\mathrm{v}_{\mathrm{i}} \mathrm{v}_{\mathrm{i}+1}\right)=6 \mathrm{i}+7,1 \leq \mathrm{i} \leq 3 ; \phi^{*}\left(\mathrm{v}_{\mathrm{i}} \mathrm{v}_{\mathrm{i}+1}\right)=9 \mathrm{i}-5,4 \leq \mathrm{i} \leq \mathrm{m}-1 ; \phi^{*}\left(\mathrm{u}_{1} \mathrm{v}_{1}\right)=7, \phi^{*}\left(\mathrm{u}_{2} \mathrm{v}_{2}\right)=10$, $\phi^{*}\left(\mathrm{u}_{3} \mathrm{v}_{3}\right)=16, \phi^{*}\left(\mathrm{u}_{\mathrm{i}} \mathrm{v}_{\mathrm{i}}\right)=9 \mathrm{i}-8,4 \leq \mathrm{i} \leq \mathrm{m} ; \phi^{*}\left(\mathrm{~s}_{\mathrm{i}} \mathrm{t}_{\mathrm{i}}\right)=9 \mathrm{~m}+6 \mathrm{i}-11,1 \leq \mathrm{i} \leq \mathrm{n} ; \phi^{*}\left(\mathrm{~s}_{\mathrm{i}} \mathrm{s}_{\mathrm{i}+1}\right)=9 \mathrm{~m}+6 \mathrm{i}-8$, $1 \leq \mathrm{i} \leq \mathrm{n}-1$. Thus the edges get distinct labels $1,4, \ldots, 3 \mathrm{q}-2$. In this case $\phi$ is a one modulo three root square mean labeling for G. Hence $L_{m} \cup\left(P_{n} \odot K_{l}\right)$ is a one modulo three root square mean graph.

Example 2.10. A one modulo three root square mean labeling of $L_{2} \cup\left(P_{4} \odot K_{1}\right)$ and $L_{7}$ $\mathrm{U}\left(P_{6} \odot K_{1}\right)$ are shown in figure 7 and figure 8 respectively.
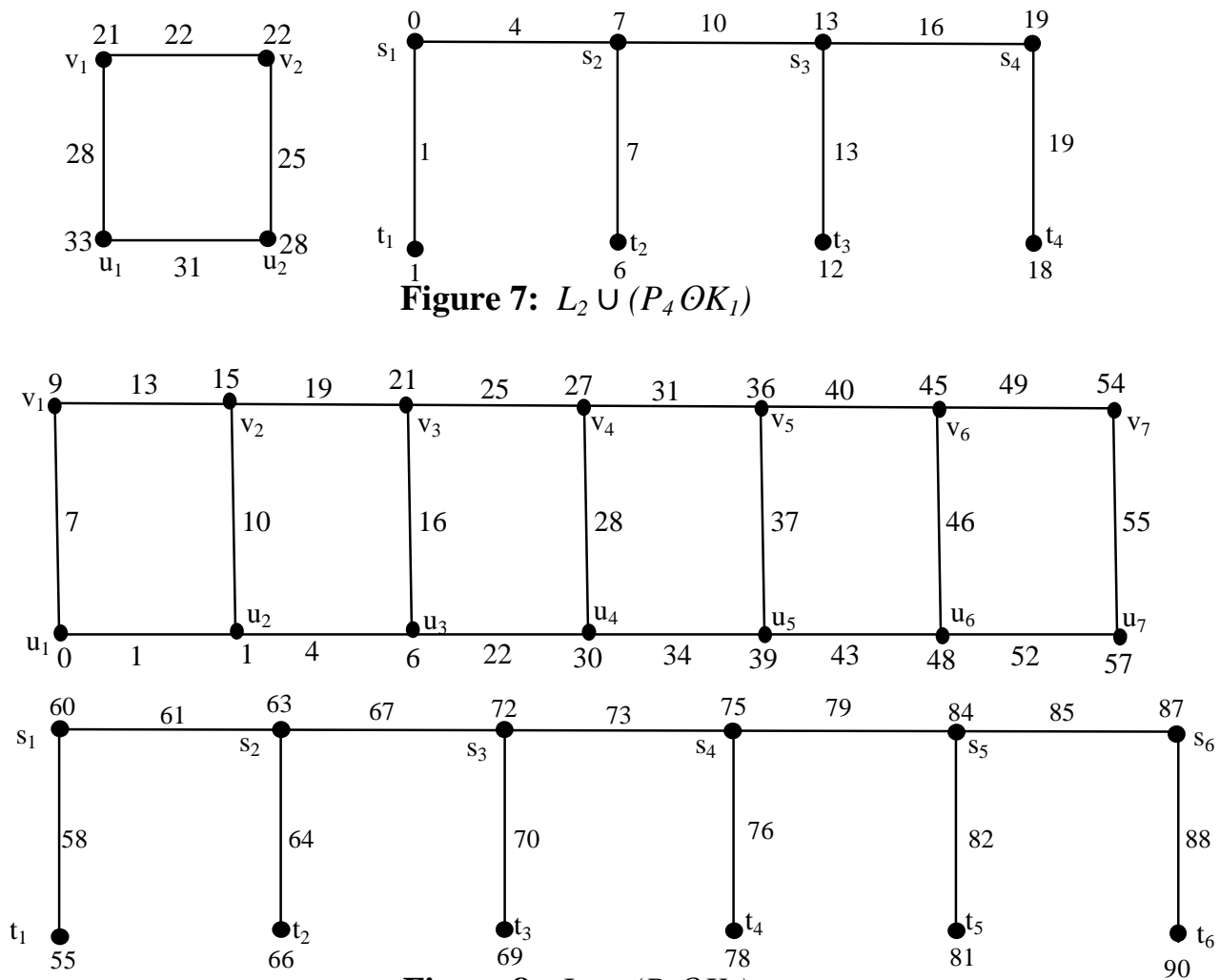

Figure 8: $L_{7} \cup\left(P_{6} \odot K_{1}\right)$ 


\section{Jaslin Melbha and C. Jayasekaran}

Theorem 2.11. $\left(\mathrm{P}_{\mathrm{m}} \odot \bar{K}_{3}\right) \cup P_{n}$ is a one modulo three root square mean graph.

Proof: Let $\mathrm{u}_{1} \mathrm{u}_{2} \ldots . \mathrm{u}_{\mathrm{m}}$ be the path $\mathrm{P}_{\mathrm{m}}$. Let $\mathrm{v}_{\mathrm{i}}, \mathrm{x}_{\mathrm{i}}, \mathrm{y}_{\mathrm{i}}, \mathrm{z}_{\mathrm{i}}$ be the vertices of $\mathrm{i}^{\text {th }}$ copy of $K_{1,3}$ with central vertex $\mathrm{v}_{\mathrm{i}}$. Identify $\mathrm{v}_{\mathrm{i}}$ with $\mathrm{u}_{\mathrm{i}}, 1 \leq \mathrm{i} \leq \mathrm{m}$. Let $\mathrm{w}_{1} \mathrm{w}_{2} \ldots \mathrm{w}_{\mathrm{n}}$ be the path $\mathrm{P}_{\mathrm{n}}$. The resultant graph is $G=\left(\mathrm{P}_{\mathrm{m}} \odot \bar{K}_{3}\right) \cup P_{n}$ with $V(G)=\left\{\mathrm{u}_{\mathrm{i}}, \mathrm{x}_{\mathrm{i}}, \mathrm{y}_{\mathrm{i}}, \mathrm{z}_{\mathrm{i}}, \mathrm{w}_{\mathrm{j}} / 1 \leq \mathrm{i} \leq \mathrm{m}, 1 \leq \mathrm{j} \leq \mathrm{n}\right\}$ and $E(G)=\left\{\mathrm{u}_{\mathrm{i}} \mathrm{x}_{\mathrm{i}}, \mathrm{u}_{\mathrm{i}} \mathrm{y}_{\mathrm{i}}, \mathrm{u}_{\mathrm{i}} \mathrm{z}_{\mathrm{i}}, \mathrm{u}_{\mathrm{j}} \mathrm{u}_{\mathrm{j}+1}, \mathrm{w}_{\mathrm{j}} \mathrm{w}_{\mathrm{j}+1} / 1 \leq \mathrm{i} \leq \mathrm{m}, 1 \leq \mathrm{j} \leq \mathrm{n}-1\right\}$. Then $\mathrm{G}$ has $4 \mathrm{~m}+\mathrm{n}$ vertices and $4 \mathrm{~m}+\mathrm{n}-2$ edges. Define a function $\phi: V(G) \rightarrow\{0,1,3, \ldots, 3 q-2,3 q\}$ by $\phi\left(\mathrm{u}_{1}\right)=1 ; \phi\left(\mathrm{u}_{2}\right)=$ $15 ; \phi\left(\mathrm{u}_{\mathrm{i}}\right)=12 \mathrm{i}-8,3 \leq \mathrm{i} \leq \mathrm{m} ; \phi\left(\mathrm{x}_{1}\right)=0 ; \phi\left(\mathrm{x}_{\mathrm{i}}\right)=12 \mathrm{i}-14,2 \leq \mathrm{i} \leq \mathrm{m} ; \phi\left(\mathrm{y}_{1}\right)=6 ; \phi\left(\mathrm{y}_{2}\right)=16 ; \phi\left(\mathrm{y}_{\mathrm{i}}\right)=$ $12 \mathrm{i}-9,3 \leq \mathrm{i} \leq \mathrm{m} ; \phi\left(\mathrm{z}_{\mathrm{i}}\right)=12 \mathrm{i}-3,1 \leq \mathrm{i} \leq \mathrm{m} ; \phi\left(\mathrm{w}_{1}\right)=12 \mathrm{~m}-5, \phi\left(\mathrm{w}_{\mathrm{i}}\right)=12 \mathrm{~m}+3 \mathrm{i}-6,2 \leq \mathrm{i} \leq \mathrm{n}$. Then $\phi$ induces a bijection $\phi^{*}: E(G) \rightarrow\{1,4, \ldots, 3 q-2\}$, where $\phi^{*}\left(\mathrm{u}_{\mathrm{i}} \mathrm{u}_{\mathrm{i}+1}\right)=12 \mathrm{i}-2,1 \leq \mathrm{i} \leq \mathrm{m}-1$; $\phi^{*}\left(\mathrm{u}_{\mathrm{i}} \mathrm{x}_{\mathrm{i}}\right)=12 \mathrm{i}-11,1 \leq \mathrm{i} \leq \mathrm{m} ; \phi^{*}\left(\mathrm{u}_{\mathrm{i}} \mathrm{y}_{\mathrm{i}}\right)=12 \mathrm{i}-8,1 \leq \mathrm{i} \leq \mathrm{m} ; \phi^{*}\left(\mathrm{u}_{\mathrm{i}} \mathrm{z}_{\mathrm{i}}\right)=12 \mathrm{i}-5,1 \leq \mathrm{i} \leq \mathrm{m} ; \phi^{*}\left(\mathrm{w}_{\mathrm{i}} \mathrm{w}_{\mathrm{i}+1}\right)$ $=12 \mathrm{~m}+3 \mathrm{i}-5,1 \leq \mathrm{i} \leq \mathrm{n}-1$. Therefore, $\phi$ is a one modulo three root square mean labeling. Hence $\left(\mathrm{P}_{\mathrm{m}} \odot \bar{K}_{3}\right) \cup P_{n}$ is a one modulo three root square mean graph.

Example 2.12. One modulo three root square mean labeling of $\left(\mathrm{P}_{5} \odot \bar{K}_{3}\right) \cup P_{7}$ is given in figure 6 .

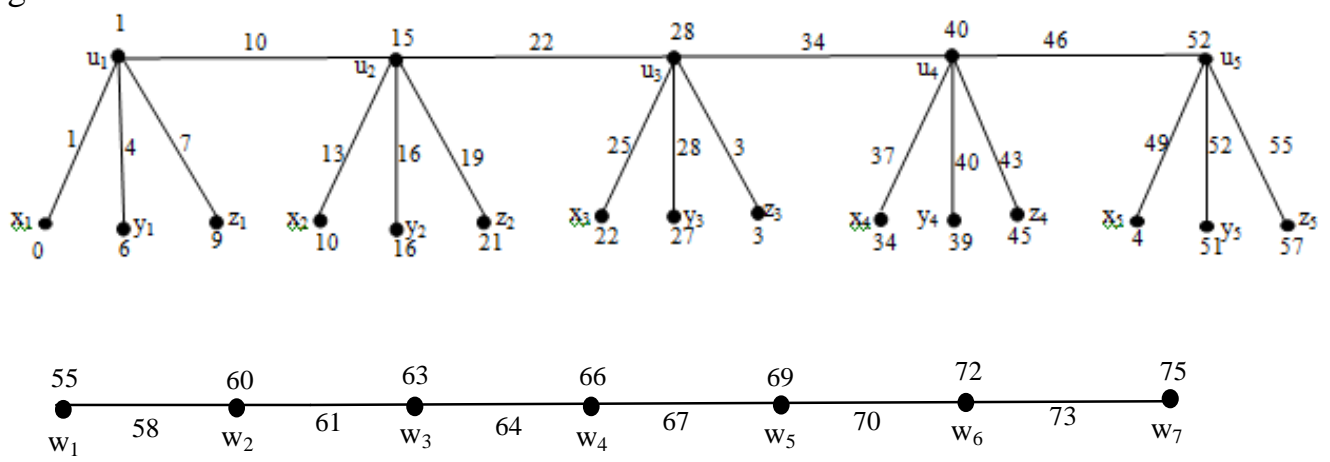

Figure 9: $\left(\mathrm{P}_{5} \odot \bar{K}_{3}\right) \cup P_{7}$

\section{Conclusion}

In this paper, we prove that some disconnected graphs $\left(L_{m} \odot K_{l}\right) \cup P_{n}$, $\left(L_{m}\right.$ $\left.\odot K_{l}\right) \cup\left(P_{n} \odot K_{l}\right), P_{n} \cup P_{m}, L_{m} \cup\left(P_{n} \odot K_{l}\right),\left(\mathrm{P}_{\mathrm{m}} \odot \bar{K}_{3}\right) \cup P_{n}$ and $L_{m} \cup P_{n}$, for $\mathrm{m} \neq 2$ and $\mathrm{n} \neq 3$ are one modulo three root square mean labeling.

Acknowledgement. The authors would like to thank reviewer(s) of this article for the time they spent and for their valuable suggestions which improved the presentation of the work.

\section{REFERENCES}

1. F. Harary, Graph theory, Narosa Publishing House, New Delhi (1998).

2. J.A.Gallian, A dynamic survey of graph labeling, The Electronics Journal of Combinatorics, 17 (2014).

3. M.Pal, Intersection graphs: An introduction, Annals of Pure and Applied Mathematics, 4 (1) (2013) 41 - 93.

4. A.Saha, M.Pal and T.K.Pal, Selection of programme slots of television channels for giving advertisement: A graph theoretic approach, Information Sciences, 177 (12) (2007) $2480-2492$. 
One Modulo Three Root Square Mean Labeling of Some Disconnected Graphs

5. C.David Raj and C.Jayasekaran, Some results on one modulo three harmonic mean graphs, International Journal of Mathematical Archieve, 5 (2014) 203-208.

6. S.S.Sandhya, S.Somasundaram and S.Anusa, Root square mean labeling of graphs, International Journal of Contemporary Mathematical Sciences, 9 (2014) 667-676.

7. C.Jayasekaran and M. Jaslin Melbha, One modulo three root square mean labeling of path related graphs, to appear in International Journal of Pure and Applied Mathematics.

8. C.Jayasekaran and M. Jaslin Melbha, Some more results on one modulo three root square mean labeling of graphs, Communicated. 